\title{
First Forcer results on deep-inelastic scattering and related quantities
}

\author{
B. Ruijl, T. Ueda, J.A.M. Vermaseren
}

Nikhef Theory Group, Science Park 105, 1098 XG Amsterdam, The Netherlands

E-mails: benrl@nikhef.nl, tueda@nikhef.nl, t68@nikhef.nl

\section{J. Davies, A. Vogt}

Department of Mathematical Sciences, University of Liverpool, Liverpool L69 3BX, UK

E-mails: Joshua.Davies@liv.ac.uk, Andreas.Vogteliv.ac.uk

\begin{abstract}
We present results on the fourth-order splitting functions and coefficient functions obtained using FORCER, a four-loop generalization of the MINCER program for the parametric reduction of selfenergy integrals. We have computed the respective lowest three even- $N$ and odd- $N$ moments for the non-singlet splitting functions and the non-singlet coefficient functions in electromagnetic and $v+\bar{v}$ charged-current deep-inelastic scattering, and the $N=2$ and $N=4$ results for the corresponding flavour-singlet quantities. Enough moments have been obtained for an LLL-based determination of the analytic $N$-dependence of the $n_{f}^{3}$ and $n_{f}^{2}$ parts, respectively, of the singlet and non-singlet splitting functions. The large- $N$ limit of the latter provides the complete $n_{f}^{2}$ contributions to the four-loop cusp anomalous dimension. Our results also provide additional evidence of a non-vanishing contribution of quartic group invariants to the cusp anomalous dimension.
\end{abstract}

Loops and Legs in Quantum Field Theory

24-29 April 2016, Leipzig, Germany

*also at Leiden Centre of Data Science, Leiden University, Niels Bohrweg 1, 2333 CA Leiden, The Netherlands.

† Speaker. 


\section{Introduction}

Impressive progress has been made in the past years on turning the next-to-next-to-leading order (NNLO, $\mathrm{N}^{2} \mathrm{LO}$ ) of perturbative QCD into the new default approximation for many hard processes, see, e.g., Refs. [1] for some very recent calculations. While this accuracy is fully adequate for most quantities, there are cases where the next order, $\mathrm{N}^{3} \mathrm{LO}$, is of interest due to (a) very high requirements on the theoretical accuracy, such as in the determination of the strong coupling constant $\alpha_{\mathrm{s}}$ from deep-inelastic scattering (DIS), see, e.g., Ref. [2], or (b) a slow convergence of the perturbation series, such as for Higgs production in proton-proton collisions, see, e.g., Refs. [3].

$\mathrm{N}^{3} \mathrm{LO}$ analyses of processes with initial-state hadrons require, in principle, parton distributions $f_{i}\left(x, \mu^{2}\right)$ determined at the same accuracy, including the renormalization-group dependence

$$
\frac{d}{d \ln \mu^{2}} f_{i}\left(x, \mu^{2}\right)=\sum_{k}\left[P_{i k}\left(\alpha_{\mathrm{s}}\left(\mu^{2}\right)\right)_{\mathrm{N}^{3} \mathrm{LO}} \otimes f_{k}\left(\mu^{2}\right)\right](x)
$$

on the factorization and renormalization scale $\mu \equiv \mu_{F}=\mu_{R}$ with the splitting functions

$$
P_{i k}\left(x, \alpha_{\mathrm{s}}\right)_{\mathrm{N}^{3} \mathrm{LO}}=\alpha_{\mathrm{s}} P_{i k}^{(0)}(x)+\alpha_{\mathrm{s}}^{2} P_{i k}^{(1)}(x)+\alpha_{\mathrm{s}}^{3} P_{i k}^{(2)}(x)+\alpha_{\mathrm{s}}^{4} P_{i k}^{(3)}(x) .
$$

Here $\otimes$ represents the Mellin convolution in the momentum fractions $x$, and the sum over $k$ includes all $n_{f}$ effectively massless quark flavours; i.e., Eq. (1.1) is a system of $\left(2 n_{f}+1\right) \times\left(2 n_{f}+1\right)$ coupled integro-differential equations. The splitting functions at NNLO [4] suggest that the effect of the $\alpha_{\mathrm{s}}^{4}$ corrections in Eq. (1.1) is very small at $x \gtrsim 10^{-2}$, but this expectation cannot be extended with sufficient certainty to the full range of $x$ probed by benchmark processes at the LHC.

Here we report on the first steps of a project that aims to obtain a phenomenologically relevant amount of information on all functions $P_{i k}^{(3)}(x)$ in Eq. (1.2). The idea is to employ the FORCER program, see Ref. [5], to extend the MINCER-based [6] fixed Mellin- $N$ calculations of Refs. [7] to four-loop accuracy, and then to construct approximate $x$-space expressions, analogous to those for $P_{i k}^{(2)}(x)$ in Refs. [8], from these results and information about the small- $x$ and large- $x$ limits [9-15].

Using basic symmetries, the system (1.1) can be decomposed into $2 n_{f}-1$ scalar equations and a $2 \times 2$ flavour-singlet system. The former (non-singlet) part consists of the $2\left(n_{f}-1\right)$ flavour asymmetries of quark-antiquark sums and differences, $q_{i} \pm \bar{q}_{i}$, and the total valence distribution,

$$
q_{\mathrm{ns}, i k}^{ \pm}=q_{i} \pm \bar{q}_{i}-\left(q_{k} \pm \bar{q}_{k}\right), \quad q_{\mathrm{ns}}^{\mathrm{v}}=\sum_{r=1}^{n_{f}}\left(q_{r}-\bar{q}_{r}\right)
$$

with

$$
P_{\mathrm{ns}}^{ \pm}=P_{\mathrm{qq}}^{\mathrm{v}} \pm P_{\mathrm{q} \overline{\mathrm{q}}}^{\mathrm{v}}, \quad P_{\mathrm{ns}}^{\mathrm{v}}=P_{\mathrm{qq}}^{\mathrm{v}}-P_{\mathrm{q} \overline{\mathrm{q}}}^{\mathrm{v}}+n_{f}\left(P_{\mathrm{qq}}^{\mathrm{s}}-P_{\mathrm{q} \overline{\mathrm{q}}}^{\mathrm{s}}\right) \equiv P_{\mathrm{ns}}^{-}+P_{\mathrm{ns}}^{\mathrm{s}} .
$$

Typical lowest-order diagrams for the different contributions in Eq. (1.4) are shown below.

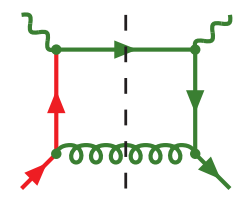

$$
P_{\mathrm{qq}}^{\mathrm{v}}=\mathscr{O}\left(\alpha_{\mathrm{s}}\right)
$$

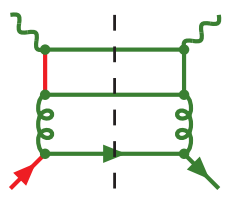

$P_{\mathrm{qq}}^{\mathrm{s}}, P_{\mathrm{q} \overline{\mathrm{q}}}^{\mathrm{s}}: \alpha_{\mathrm{s}}^{2}$

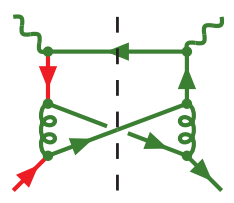

$P_{\mathrm{q} \overline{\mathrm{q}}}^{\mathrm{v}}: \alpha_{\mathrm{s}}^{2}$

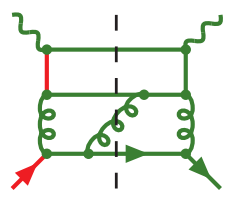

$P_{\mathrm{q} \overline{\mathrm{q}}}^{\mathrm{s}} \neq P_{\mathrm{qq}}^{\mathrm{s}}: \alpha_{\mathrm{s}}^{3}$ 
The remaining flavour-singlet quantities and their scale dependence (evolution) are given by

$$
q_{\mathrm{s}}=\sum_{r=1}^{n_{f}}\left(q_{r}+\bar{q}_{r}\right), \quad \frac{d}{d \ln \mu^{2}}\left(\begin{array}{c}
q_{\mathrm{s}} \\
g
\end{array}\right)=\left(\begin{array}{cc}
P_{\mathrm{qq}} & P_{\mathrm{qg}} \\
P_{\mathrm{gq}} & P_{\mathrm{gg}}
\end{array}\right) \otimes\left(\begin{array}{c}
q_{\mathrm{s}} \\
g
\end{array}\right)
$$

where $g\left(x, \mu^{2}\right)$, abbreviated by $g$, denotes the gluon distribution. $P_{\mathrm{qq}}$ differs from $P_{\mathrm{ns}}^{+}$in Eq. (1.4) by an additional pure singlet (ps) contribution starting at order $\alpha_{\mathrm{s}}^{2}$,

$$
P_{\mathrm{qq}}=P_{\mathrm{ns}}^{+}+n_{f}\left(P_{\mathrm{qq}}^{\mathrm{s}}+P_{\mathrm{q} \overline{\mathrm{q}}}^{\mathrm{s}}\right) \equiv P_{\mathrm{ns}}^{+}+P_{\mathrm{ps}} .
$$

Decompositions analogous to Eqs. (1.4) and (1.6) apply to the coefficient functions for inclusive DIS. In fact, following Refs. [7], our calculations of the $\mathrm{N}^{3} \mathrm{LO}$ splitting function are carried out via the unfactorized fourth-order coefficient functions in dimensional regularization, transformed to forward amplitudes $A$ using the optical theorem and projected onto the $N$-th Mellin moment,

$$
A(N)=\int_{0}^{1} d x x^{N-1} A(x),
$$

by a dispersion relation in $x$. Like the operator-product expansion, this approach determines either the even or the odd moments of the splitting and coefficient functions. Specifically, the even moments are obtained of quantities involving $q+\bar{q}$, such as $F_{2}$ and $F_{L}$ in electromagnetic and $v+\bar{v}$ charged-current DIS, and the odd moments of quantities with $q-\bar{q}$, such as $F_{3}$ in $v+\bar{v}$ chargedcurrent DIS. See Ref. [16] for a detailed discussion of these issues including the $v-\bar{v}$ cases.

Before we turn to our new results, it is worthwhile to briefly recall the large- $N$ structure of the quark-quark splitting functions in the $\overline{\mathrm{MS}}$ scheme employed throughout this article,

$$
\gamma_{\mathrm{ns}}^{(n) \pm, \mathrm{v}}(N) \equiv-P_{\mathrm{ns}}^{(n) \pm, \mathrm{v}}(N)=A_{n} \ln N-B_{n}+C_{n} N^{-1} \ln N-D_{n}+\mathscr{O}_{ \pm}\left(N^{-2}\right) .
$$

Here $A_{n}$ is the (n+1)-loop cusp anomalous dimension [12], and $C_{n>2}$ has been predicted in terms of $A_{k<n}$ in Ref. [13]. The differences between the $q q$ splitting functions are of order $N^{-2}$ at large $N$.
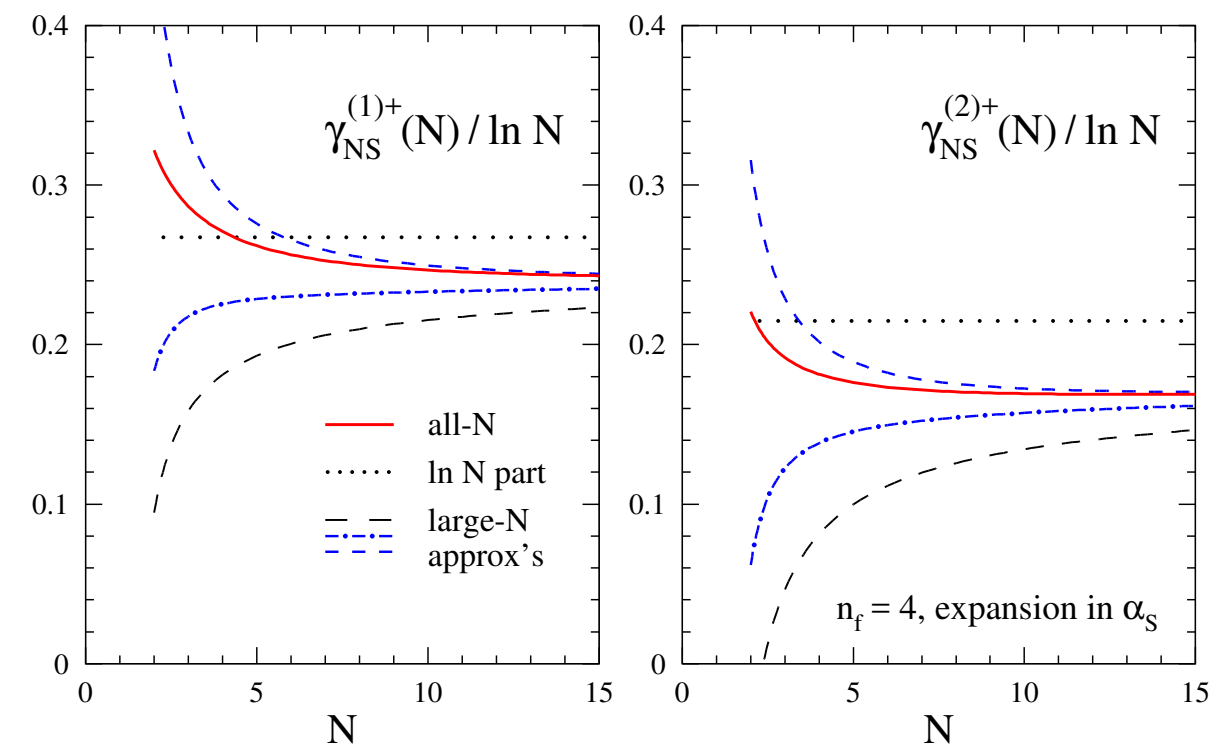

Figure 1: The successive large- $N$ approximations in Eq. 1.8) compared to the full NLO and NNLO results. 


\section{Low- $N$ results for splitting functions and coefficient functions}

As an example of our analytic results, we present the $N=4$ anomalous dimension $\gamma_{\mathrm{gg}}^{(3)}$, defined as in Eq. (1.8) above, for a general gauge group in terms of the expansion parameter $a_{\mathrm{s}} \equiv \alpha_{\mathrm{s}} /(4 \pi)$,

$$
\begin{aligned}
\gamma_{\mathrm{gg}}^{(3)} & (N=4)=C_{A}^{4}\left(\frac{1502628149}{3375000}+\frac{1146397}{11250} \zeta_{3}-\frac{504}{5} \zeta_{5}\right)+\frac{d_{A}^{a b c d} d_{A}^{a b c d}}{n_{a}}\left(\frac{21623}{150}\right. \\
& \left.+\frac{15596}{15} \zeta_{3}-\frac{6048}{5} \zeta_{5}\right)-n_{f} C_{A}^{3}\left(\frac{20580892841}{72900000}+\frac{12550223}{22500} \zeta_{3}-\frac{8613}{25} \zeta_{4}-\frac{4316}{27} \zeta_{5}\right) \\
+ & n_{f} \frac{d_{F}^{a b c d} d_{A}^{a b c d}}{n_{a}}\left(\frac{160091}{675}+\frac{80072}{225} \zeta_{3}-\frac{48016}{45} \zeta_{5}\right)-n_{f} C_{A}^{2} C_{F}\left(\frac{4212122951}{41006250}\right. \\
& \left.-\frac{1170784}{5625} \zeta_{3}+\frac{418198}{1125} \zeta_{4}-\frac{17636}{45} \zeta_{5}\right)+n_{f} C_{A} C_{F}^{2}\left(\frac{1913110089023}{26244000000}+\frac{39313783}{101250} \zeta_{3}\right. \\
& \left.+\frac{26741}{750} \zeta_{4}-\frac{3082}{5} \zeta_{5}\right)+n_{f} C_{F}^{3}\left(\frac{34764568601}{2099520000}-\frac{958343}{40500} \zeta_{3}-\frac{18997}{2250} \zeta_{4}+\frac{908}{45} \zeta_{5}\right) \\
& -n_{f}^{2} C_{A}^{2}\left(\frac{3250393649}{218700000}-\frac{2969291}{20250} \zeta_{3}+\frac{1566}{25} \zeta_{4}+\frac{1276}{135} \zeta_{5}\right)-n_{f}^{2} C_{F}^{2}\left(\frac{275622924731}{26244000000}\right. \\
& \left.-\frac{253369}{10125} \zeta_{3}+\frac{1078}{225} \zeta_{4}\right)+n_{f}^{2} C_{A} C_{F}\left(\frac{136020246173}{3280500000}-\frac{1672751}{10125} \zeta_{3}+\frac{15172}{225} \zeta_{4}\right) \\
+ & n_{f}^{2} \frac{d_{F}^{a b c d} d_{F}^{a b c d}}{n_{a}}\left(\frac{75788}{675}+\frac{3008}{15} \zeta_{3}-\frac{20416}{45} \zeta_{5}\right)+n_{f}^{3} C_{F}\left(\frac{1780699}{24300000}-\frac{484}{675} \zeta_{3}\right) \\
& -n_{f}^{3} C_{A}\left(\frac{20440457}{21870000}-\frac{1888}{405} \zeta_{3}\right) .
\end{aligned}
$$

Except for the last line [17], Eq. (2.1) is a new result. The complete set of fourth-order anomalous dimensions at $N \leq 4$ for Eq. (1.5) and at $N \leq 6$ for Eq. (1.4) will be presented elsewhere [18].

Our results for $\gamma_{\mathrm{ns}}^{(3) \pm}(N)$ agree with the calculations at $N \leq 4$ in Refs. [19-21]. The numerical size of these quantities is shown in Fig. 2 for $n_{f}=3$ and $n_{f}=4$ light flavours. Taking into account the very slow large- $N$ convergence of $\gamma_{\mathrm{ns}}^{(n)}(N) / \ln N$ to $A_{n}$ in Eq. (1.8), see Fig. 1 above, our results are consistent with, but not yet sufficient to improve on, the Padé estimate of $A_{3}$ in Ref. [22]. Similarly, the $N$-dependent Padé estimate used in $\mathrm{N}^{3} \mathrm{LO}$ determinations of $\alpha_{\mathrm{s}}$ from non-singlet DIS [2] agrees with the calculated moments well within the large uncertainty assigned to it so far.

Inserting the QCD colour factors (the quartic group invariants are normalized as in Ref. [23]), the numerical expansions of the even- $N$ non-singlet anomalous dimensions at $n_{f}=4$ are given by

$$
\begin{aligned}
& \gamma_{\mathrm{ns}}^{+}(2,4)=0.28294 \alpha_{\mathrm{s}}\left(1+0.7987 \alpha_{\mathrm{s}}+0.5451 \alpha_{\mathrm{s}}^{2}+0.5215 \alpha_{\mathrm{s}}^{3}+\ldots\right), \\
& \gamma_{\mathrm{ns}}^{+}(4,4)=0.55527 \alpha_{\mathrm{s}}\left(1+0.6851 \alpha_{\mathrm{s}}+0.4564 \alpha_{\mathrm{s}}^{2}+0.3659 \alpha_{\mathrm{s}}^{3}+\ldots\right), \\
& \gamma_{\mathrm{ns}}^{+}(6,4)=0.71645 \alpha_{\mathrm{s}}\left(1+0.6497 \alpha_{\mathrm{s}}+0.4368 \alpha_{\mathrm{s}}^{2}+0.3307 \alpha_{\mathrm{s}}^{3}+\ldots\right) .
\end{aligned}
$$

The corresponding results for the odd- $N$ cases $\gamma_{\mathrm{ns}}^{\mathrm{a}}$ for $\mathrm{a}=-, \mathrm{v}$ are $\gamma_{\mathrm{ns}}^{\mathrm{a}}\left(1, n_{f}\right)=0$, as required by fermion-number conservation, and

$$
\begin{array}{r}
\gamma_{\mathrm{ns}}^{\mathrm{a}}(3,4)=0.44210 \alpha_{\mathrm{s}}\left(1+0.7218 \alpha_{\mathrm{s}}+0.4767 \alpha_{\mathrm{s}}^{2}+0.3921 \alpha_{\mathrm{s}}^{3}+\ldots\right. \\
\left.+\delta_{\mathrm{av}}\left[0.0144 \alpha_{\mathrm{s}}^{2}+0.0045 \alpha_{\mathrm{s}}^{3}+\ldots\right]\right), \\
\gamma_{\mathrm{ns}}^{\mathrm{a}}(5,4)=0.64369 \alpha_{\mathrm{s}}\left(1+0.6636 \alpha_{\mathrm{s}}+0.4434 \alpha_{\mathrm{s}}^{2}+0.3421 \alpha_{\mathrm{s}}^{3}+\ldots\right. \\
\left.+\delta_{\mathrm{av}}\left[0.0032 \alpha_{\mathrm{s}}^{2}+0.0024 \alpha_{\mathrm{s}}^{3}+\ldots\right]\right) .
\end{array}
$$



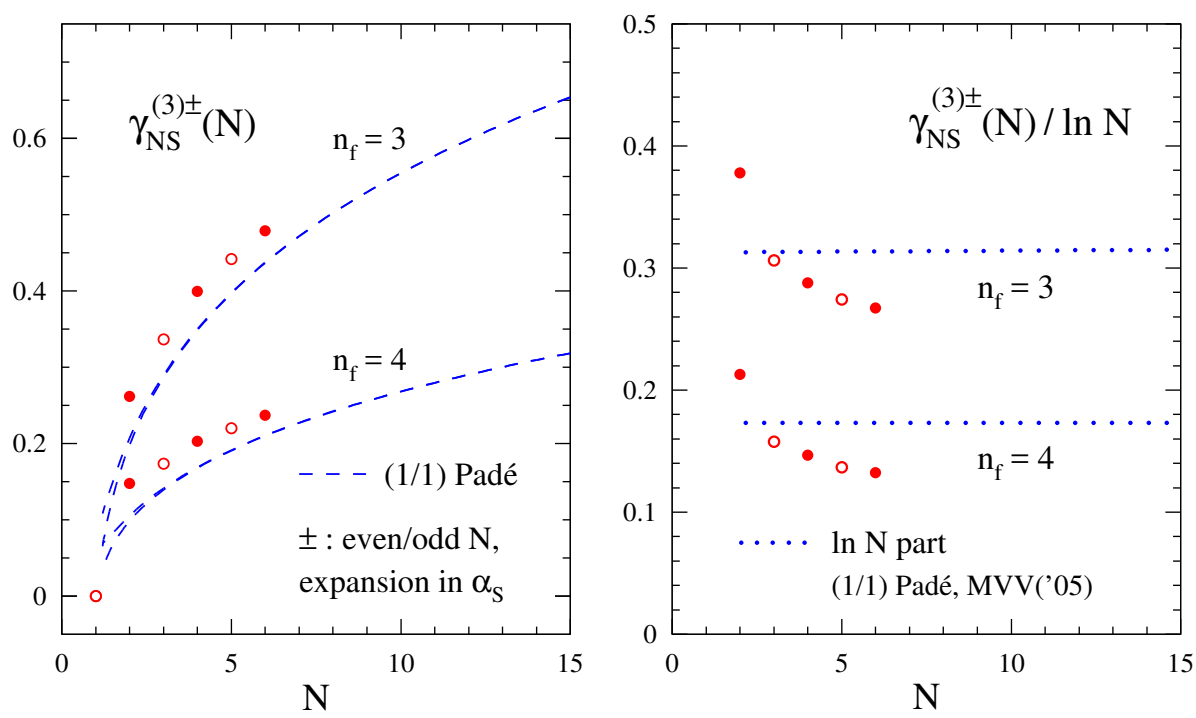

Figure 2: The lowest three even- $N$ and odd- $N$ values, respectively, of the anomalous dimensions $\gamma_{\mathrm{ns}}^{(3)+}$ and $\gamma_{\mathrm{ns}}^{(3)-}$ in Eqs. (1.4) and (1.8), compared to Padé estimates derived from the NNLO results of Ref. [4].

The first two moments of the upper row of the splitting-function matrix in Eq. (1.5) read

$$
\begin{aligned}
& \gamma_{\mathrm{qq}}(2,4)=0.28294 \alpha_{\mathrm{s}}\left(1+0.6219 \alpha_{\mathrm{s}}+0.1461 \alpha_{\mathrm{s}}^{2}+0.3662 \alpha_{\mathrm{s}}^{3}+\ldots\right), \\
& \gamma_{\mathrm{qq}}(4,4)=0.55527 \alpha_{\mathrm{s}}\left(1+0.6803 \alpha_{\mathrm{s}}+0.4278 \alpha_{\mathrm{s}}^{2}+0.3459 \alpha_{\mathrm{s}}^{3}+\ldots\right), \\
& \gamma_{\mathrm{qg}}(2,4)=-0.21221 \alpha_{\mathrm{s}}\left(1+0.9004 \alpha_{\mathrm{s}}-0.1028 \alpha_{\mathrm{s}}^{2}-0.2367 \alpha_{\mathrm{s}}^{3}+\ldots\right), \\
& \gamma_{\mathrm{qg}}(4,4)=-0.11671 \alpha_{\mathrm{s}}\left(1-0.2801 \alpha_{\mathrm{s}}-0.9986 \alpha_{\mathrm{s}}^{2}+0.1297 \alpha_{\mathrm{s}}^{3}+\ldots\right)
\end{aligned}
$$

For the lower row we find $\gamma_{\mathrm{gi}}\left(2, n_{f}\right)=-\gamma_{\mathrm{qi}}\left(2, n_{f}\right)$, as required by the momentum sum rule, and

$$
\begin{aligned}
& \gamma_{\mathrm{gq}}(4,4)=-0.07781 \alpha_{\mathrm{s}}\left(1+1.1152 \alpha_{\mathrm{s}}+0.8234 \alpha_{\mathrm{s}}^{2}+0.8833 \alpha_{\mathrm{s}}^{3}+\ldots\right) \\
& \gamma_{\mathrm{gg}}(4,4)=1.21489 \alpha_{\mathrm{s}}\left(1+0.3835 \alpha_{\mathrm{s}}+0.1220 \alpha_{\mathrm{s}}^{2}+0.2406 \alpha_{\mathrm{s}}^{3}+\ldots\right) .
\end{aligned}
$$

The relative $\mathrm{N}^{3} \mathrm{LO}$ corrections are somewhat larger for $n_{f}=3$, but are small in all cases with coefficients $\lesssim 1$ for $n_{f}=3, \ldots, 6$ in Eqs. (2.2) - 2.6), where our new results are given in blue.

A check of the matrix in Eq. (1.5) at $N>2$ is provided by a relation between the anomalous dimensions which emerges for $n_{f}=1$ Majorana quarks and the choice $C_{F}=2 T_{F}=C_{A} \equiv n_{c} \equiv$ $n_{\text {colours }}$ of the colour factors that leads to a supersymmetric theory [24]: The combination ${ }^{1}$

$$
\Delta_{\mathrm{S}}^{(n)}(N)=-\gamma_{\mathrm{qq}}^{(n)}(N)-\gamma_{\mathrm{gq}}^{(n)}(N)+\gamma_{\mathrm{qg}}^{(n)}(N)+\gamma_{\mathrm{gg}}^{(n)}(N)
$$

is supposed to vanish for a regularization that does not violate the supersymmetry. In dimensional regularization $\Delta_{\mathrm{S}}^{(n)}$ does not vanish, but is much simpler than the anomalous dimensions, see Ref. [25] for a brief discussion at NNLO. We find that this expected simplification occurs also at $\mathrm{N}^{3} \mathrm{LO}$ (at $N=4$, for now: $\Delta_{\mathrm{S}}$ vanishes at $N=2$ already in QCD due to the momentum sum rule) at

$$
\left(2 n_{f}\right)^{2} \frac{d_{F F}^{(4)}}{n_{a}}=2 n_{f} \frac{d_{F A}^{(4)}}{n_{a}}=2 n_{f} \frac{d_{F F}^{(4)}}{n_{c}}=\frac{d_{F A}^{(4)}}{n_{c}}=\frac{d_{A A}^{(4)}}{n_{a}}, \quad d_{\mathrm{xy}}^{(4)} \equiv d_{x}^{a b c d} d_{y}^{a b c d}
$$

\footnotetext{
${ }^{1} \mathrm{Up}$ to NNLO, the same results are obtained by keeping the QCD value $T_{F}=1 / 2$ and setting $n_{f}=n_{c}$.
} 
for the quartic group invariants with all particles in the adjoint representation. The additional factor of two for each power of $n_{f}$ in the QCD results is due to the transition to Majorana fermions.

We now briefly turn to the coefficient functions $C_{a}$ for DIS in massless perturbative QCD [26]; see Refs. [27] for the important heavy-quark contributions. The size of the fourth-order corrections is illustrated in Fig. 3 for the structure functions $F_{2, \text { ns }}, F_{3}$ and $F_{L, \text { ns }}$ in charged-current $v+\bar{v}$ DIS. For $F_{2}$ and $F_{3}$ the $\ln ^{8} N \ldots \ln ^{2} N$ large- $N$ contributions to $C_{a, q}^{(4)}(N)$ are fixed by the softgluon exponentiation [22], and the subleading $N^{-1}\left(\ln ^{7} N \ldots \ln ^{4} N\right)$ terms by the double-logarithmic resummations in Refs. [14,28]. For $C_{L, q}^{(4)}(N)$ the latter provide the $N^{-1}\left(\ln ^{6} N \ldots \ln ^{4} N\right)$ contributions.
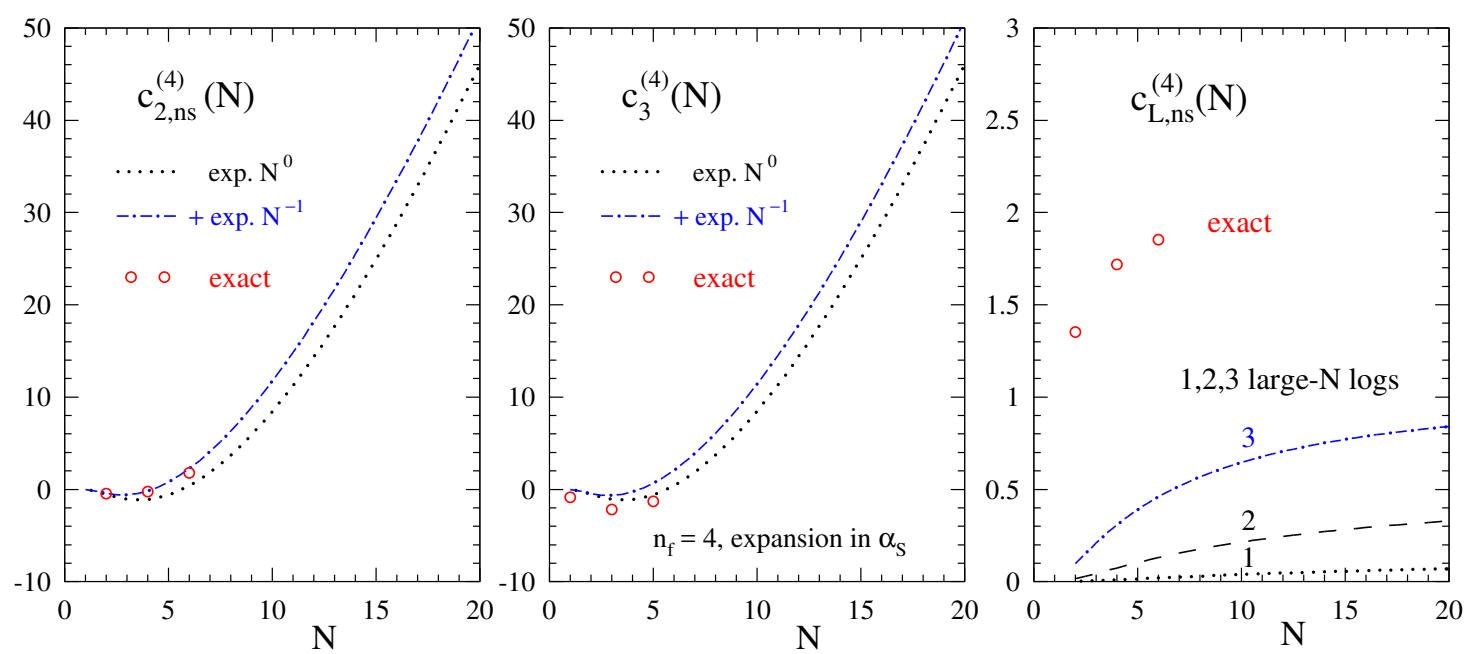

Figure 3: The moments calculated so far of the fourth-order coefficient functions $c_{2, \mathrm{~ns}}^{(4)}, c_{3}^{(4)}$ and $c_{L, \mathrm{~ns}}^{(4)}$ for $v+\bar{v}$ charged-current DIS at $n_{f}=4$. Also show are the contributions provided by large- $N$ resummations.

The numerical $\alpha_{\mathrm{s}}$ expansions of these coefficient functions at low values of $N$ read, for $n_{f}=4$,

$$
\begin{array}{r}
C_{2, \mathrm{~ns}}(2,4)=1+0.0354 \alpha_{\mathrm{s}}-0.0231 \alpha_{\mathrm{s}}^{2}-0.0613 \alpha_{\mathrm{s}}^{3}-0.4746 \alpha_{\mathrm{s}}^{4}+\ldots, \\
C_{2, \mathrm{~ns}}(4,4)=1+0.4828 \alpha_{\mathrm{s}}+0.4711 \alpha_{\mathrm{s}}^{2}+0.4727 \alpha_{\mathrm{s}}^{3}-0.2458 \alpha_{\mathrm{s}}^{4}+\ldots, \\
C_{2, \mathrm{~ns}}(6,4)=1+0.8894 \alpha_{\mathrm{s}}+1.2053 \alpha_{\mathrm{s}}^{2}+1.7571 \alpha_{\mathrm{s}}^{3}+1.7748 \alpha_{\mathrm{s}}^{4}+\ldots, \\
C_{3, \mathrm{~ns}}(1,4)=1-0.3183 \alpha_{\mathrm{s}}-0.3293 \alpha_{\mathrm{s}}^{2}-0.4467 \alpha_{\mathrm{s}}^{3}-1.0512 \alpha_{\mathrm{s}}^{4}+\ldots \\
+\delta_{\mathrm{av}}\left[0.0533 \alpha_{\mathrm{s}}^{3}+0.1999 \alpha_{\mathrm{s}}^{4}+\ldots\right], \\
C_{3, \mathrm{~ns}}(3,4)=1+0.1326 \alpha_{\mathrm{s}}-0.0852 \alpha_{\mathrm{s}}^{2}-0.5202 \alpha_{\mathrm{s}}^{3}-2.2510 \alpha_{\mathrm{s}}^{4}+\ldots \\
+\delta_{\mathrm{av}}\left[0.0202 \alpha_{\mathrm{s}}^{3}+0.0805 \alpha_{\mathrm{s}}^{4}+\ldots\right], \\
C_{3, \mathrm{~ns}}(5,4)=1+0.6166 \alpha_{\mathrm{s}}+0.6042 \alpha_{\mathrm{s}}^{2}+0.4214 \alpha_{\mathrm{s}}^{3}-1.3217 \alpha_{\mathrm{s}}^{4}+\ldots \\
+\delta_{\mathrm{av}}\left[0.00788 \alpha_{\mathrm{s}}^{3}+0.0422 \alpha_{\mathrm{s}}^{4}+\ldots\right], \\
C_{L, \mathrm{~ns}}(2,4)=0.14147 \alpha_{\mathrm{s}}\left(1+1.7270 \alpha_{\mathrm{s}}+3.7336 \alpha_{\mathrm{s}}^{2}+9.5619 \alpha_{\mathrm{s}}^{3}+\ldots\right), \\
C_{L, \mathrm{~ns}}(4,4)=0.08488 \alpha_{\mathrm{s}}\left(1+2.5619 \alpha_{\mathrm{s}}+6.9208 \alpha_{\mathrm{s}}^{2}+20.251 \alpha_{\mathrm{s}}^{3}+\ldots\right), \\
C_{L, \mathrm{~ns}}(6,4)=0.06063 \alpha_{\mathrm{s}}\left(1+3.1557 \alpha_{\mathrm{s}}+9.6370 \alpha_{\mathrm{s}}^{2}+30.572 \alpha_{\mathrm{s}}^{3}+\ldots\right) .
\end{array}
$$

The first moment of $F_{3}$ is the Gross-Llewellyn-Smith (GLS) sum rule; its coefficients in Eq. (2.10) agree with those of Refs. [29], where the $\delta_{\text {av }}$ part [cf. Eq. (2.3)] is called the singlet contribution. 


\section{Large- $n_{f}$ all- $N$ parts of $\mathbf{N}^{3} \mathrm{LO}$ splitting functions \& the cusp anomalous dimension}

The extension of our above results to higher values of $N$ will require very considerable computing resources and further optimizations of our programs. The situation is more favourable for some leading and subleading large- $n_{f}$ contributions, which do not involve the hardest diagram topologies. For example, the top-level diagrams contributing to the $n_{f}^{2}$ parts of the anomalous dimensions $\gamma_{\mathrm{ns}}^{(3) \pm}$ are the same as for the $n_{f}$ parts of the NNLO contributions $\gamma_{\mathrm{ns}}^{(2) \pm}$ in Ref. [30],

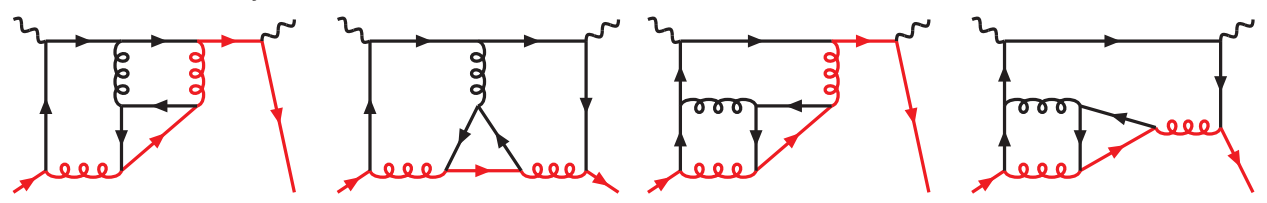

but with an additional quark loop inserted into one of the gluon propagators. These diagrams have the colour factor $C_{F} C_{A} n_{f}^{2}$; the $C_{F}^{2} n_{f}^{2}$ cases are even simpler, as is the $C_{F} n_{f}^{3}$ part derived in Ref. [31].

It is convenient to write the colour-factor decomposition the $n_{f}^{2}$ parts of $\gamma_{\mathrm{ns}}^{(3) \pm}$ in two ways,

$$
\begin{aligned}
\left.\gamma_{\mathrm{ns}}^{(3) \pm}(N)\right|_{n_{f}^{2}} & =C_{F} n_{f}^{2}\left\{C_{F} 2 A(N)+\left(C_{A}-2 C_{F}\right) B_{ \pm}(N)\right\} \\
& =C_{F} n_{f}^{2}\left\{C_{F}\left(2 A(N)-2 B_{ \pm}(N)\right)+C_{A} B_{ \pm}(N)\right\}
\end{aligned}
$$

$A(N)$ is the large- $n_{c}$ part; it is the same for the even- $N(+)$ and odd- $N(-)$ cases and should include only non-alternating harmonic sums [32]. Once $A(N)$ is known, it is possible to determine $B_{+}(N)$ and $B_{-}(N)$ from the $C_{F}$ parts in the second line of Eq. (3.1) which require only two-loop diagrams.

We have computed the even and odd moments up to $N=20$ for the determination of $A(N)$, and the even- $N$ or odd- $N$ moments up to $N=42$ for $B_{+}(N)$ and $B_{-}(N)$, respectively. These calculations are sufficient to determine all three function using an LLL-based program [33], see also Refs. [34], with a sufficient number of validation constraints. The resulting large $-n_{c}$ contribution reads

$$
\begin{aligned}
& \left.\gamma_{\mathrm{ns}}^{(3)}(N)\right|_{C_{F} n_{c} n_{f}^{2}}=\frac{127}{18}+\frac{1}{81}\left(\frac{20681}{2} \eta+2119 S_{1}-2275 \eta^{2}-20460 D_{1}^{2}+3392 S_{1} \eta-5036 S_{2}\right) \\
& \quad+\frac{4}{81}\left(118 \eta^{3}-886 D_{1}^{3}-914 S_{1} \eta^{2}-848 S_{1} D_{1}^{2}-152 S_{1,2}-416 S_{2} \eta-152 S_{2,1}+1148 S_{3}\right) \\
& \quad+\frac{8}{27}\left(-57 D_{1}^{4}+18 S_{1} \eta^{3}-24 S_{1} D_{1}^{3}+2 S_{2} \eta^{2}+128 S_{2} D_{1}^{2}-8 S_{3} \eta+40 S_{1,3}+80 S_{2,2}\right. \\
& \left.\quad+120 S_{3,1}-159 S_{4}\right)+\frac{8}{9}\left(-6 \eta^{5}-12 D_{1}^{5}+10 S_{1} \eta^{4}-24 S_{1} D_{1}^{4}+8 S_{2} \eta^{3}+4 S_{3} \eta^{2}\right. \\
& \left.\quad-8 S_{3} D_{1}^{2}+4 S_{3,1} \eta-8 S_{1,3,1}+4 S_{1,4}-8 S_{2,3}-16 S_{3,2}-2 S_{4} \eta-20 S_{4,1}+24 S_{5}\right) \\
& \quad+\zeta_{3}\left\{-\frac{44}{3}-\frac{160}{9}\left(\eta-2 S_{1}\right)+\frac{16}{3}\left(\eta^{2}-2 D_{1}^{2}-2 S_{2}\right)\right\}+\zeta_{4}\left\{12+8 \eta-16 S_{1}\right\}
\end{aligned}
$$

where all sums are taken at $N$ and we have used the abbreviations $D_{i}=(N+i)^{-1}$ and $\eta=D_{0}-D_{1}$.

The large- $N$ limit (1.8) of Eq. (3.2), together with the corresponding expressions for $B_{ \pm}(N)$ [35] yields the complete $n_{f}^{2}$ contribution to the four-loop quark cusp anomalous dimension,

$$
\begin{aligned}
\gamma_{\text {cusp }}^{(3)}= & \ldots+C_{F} C_{A} n_{f}^{2}\left(\frac{923}{81}-\frac{608}{81} \zeta_{2}+\frac{2240}{27} \zeta_{3}-\frac{112}{3} \zeta_{4}\right) \\
& +C_{F}^{2} n_{f}^{2}\left(\frac{2392}{81}-\frac{640}{9} \zeta_{3}+32 \zeta_{4}\right)-C_{F} n_{f}^{3}\left(\frac{32}{81}-\frac{64}{27} \zeta_{3}\right) .
\end{aligned}
$$



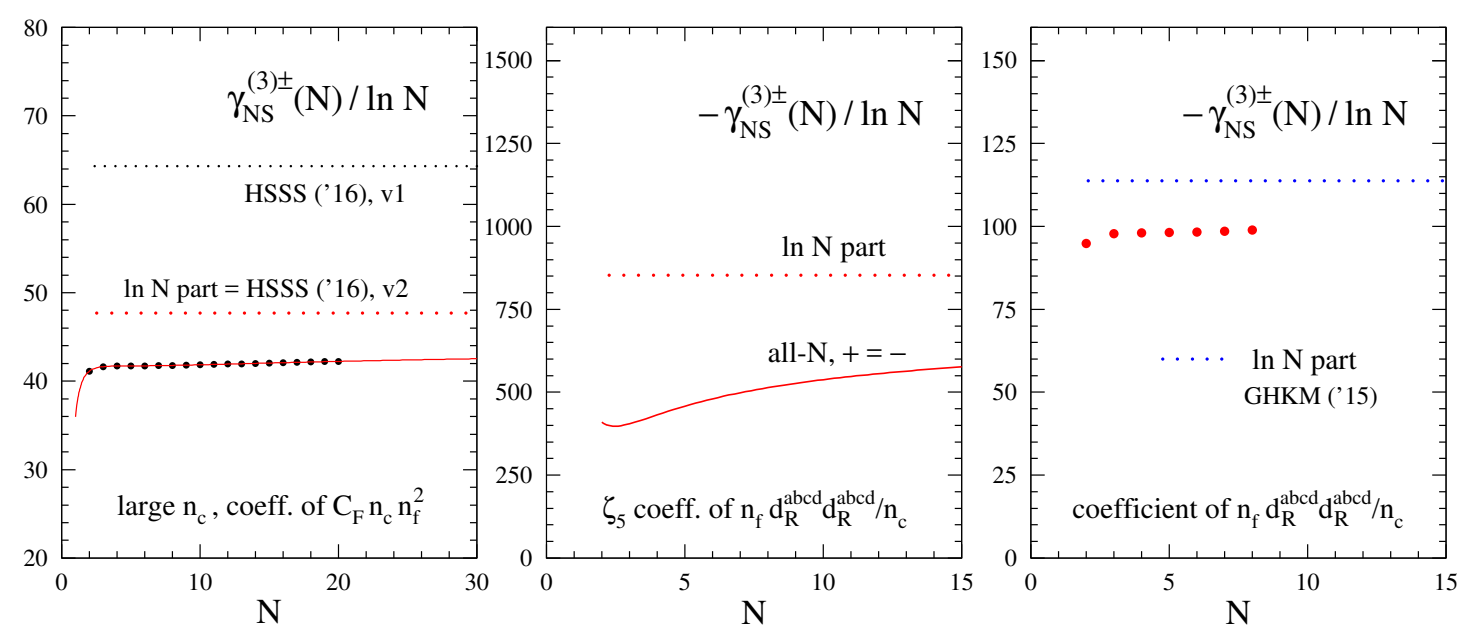

Figure 4: Fermionic contributions to the $\mathrm{N}^{3} \mathrm{LO}$ anomalous dimensions $\gamma^{(3) \pm}(N)$, compared to their calculated (left two panels) and conjectured (right panel) large- $N$ limits given by the respective parts of $\gamma_{\text {cusp }}^{(3)}$.

The large- $n_{c}$ limit of Eq. (3.3) agrees with the second version of Ref. [36], in which an error has been fixed after we pointed out a discrepancy with our result, see the left part of Fig. 4. The $C_{F}^{2} n_{f}^{2}$ coefficient agrees with the result of Ref. [37], which was converted to our notation and compared during this conference. The $n_{f}^{3}$ part of $\gamma_{\mathrm{ns}}^{(3)}(N)$, and hence the $n_{f}^{3}$ coefficient in Eq. (3.3), agrees with Ref. [31]. Our results also agree with the prediction of the $N^{-1} \ln N$ coefficient in Ref. [13] and the small- $x$ resummation result for the $1 / N^{5}$ contribution [11].

In the flavour singlet case, at least for the time being, only the $n_{f}^{3}$ leading large- $n_{f}$ contributions can be determined in this manner; the results will be presented in Ref. [35].

The question of whether or not the quartic group invariants contribute to the four-loop cusp anomalous dimension has attracted some interest, see, e.g., Refs. [38]. The presence of such contributions would violate the Casimir scaling, $\gamma_{\text {cusp }, \mathrm{q}}=C_{F} / C_{A} \gamma_{\text {cusp,g }}$, observed up to NNLO [4]. In our calculations, the relatively easiest contribution of this type is the $n_{f} d_{F}^{a b c d} d_{F}^{a b c d} / n_{c}$ part of the quark case, which appears as the corresponding $\ln N$ coefficient of $\gamma_{\mathrm{ns}}^{(3)}(N)$. So far we have extended the calculation of this contribution to $N=8$. Except for the $\zeta_{5}$ part,

$$
\left.\gamma_{\mathrm{ns}}^{(3)}(N)\right|_{\zeta_{5} n_{f} d_{F F}^{(4)} / n_{c}}=\frac{1280}{3}\left[2 S_{1}(N)-3+17\left(\frac{1}{N}-\frac{1}{N+1}\right)-6\left(\frac{1}{N^{2}}+\frac{1}{(N+1)^{2}}\right)\right]
$$

(the corresponding result in Ref. [21] is unfortunately incorrect - only four moments were available there, and the $1 / N^{2}$ and $1 /(N+1)^{2}$ contributions were erroneously assumed to be absent) this is not sufficient for a determination of the all- $N$ result from which $\gamma_{\text {cusp }}^{(3)}$ can be read off. Together with the prime content of the denominators of the calculated moments, Eq. (3.4) is suggestive, but not a positive proof, of a non-vanishing $\zeta_{5} n_{f} d_{F}^{a b c d} d_{F}^{a b c d} / n_{c}$ to $\gamma_{\text {cusp }}^{(3)}$. Moreover the calculated moments, shown in Fig. 4, clearly point to a non-vanishing value; in particular, they are consistent with the numerical value proposed in Ref. [37] on the basis of a conjectured relation to the quark-antiquark potential calculated in Refs. [39] for this colour factor. 


\section{Summary and outlook}

We have presented the first computations of anomalous dimensions and coefficient functions at order $\alpha_{\mathrm{s}}^{4}$ with FORCER, a new FORM [40] program for the analytic evaluation of four-loop massless propagator integrals. Our results agree with those of all comparable calculations performed so far. Together with the calculation of the four-loop gluon propagator in the background gauge to all powers of the gauge parameter, this provides a robust validation of the FORCER package.

So far we have extended previous calculations [19-21] of the non-singlet splitting functions for the evolution of the parton distributions of the proton by one moment each for $P_{\mathrm{ns}}^{(3)+}$ and $P_{\mathrm{ns}}^{(3)-}$. We have performed the first calculations, at $N=2$ and $N=4$, of the corresponding flavour-singlet quantities, and the first calculations of fourth-order coefficient functions in DIS beyond $N=1$ [29]. The full results will be presented in Ref. [18], together with the four-loop contributions to the renormalization factors $Z_{5}$ and $Z_{A}$ required if the Larin scheme for $\gamma_{5}[41]$ is used in the calculations.

Unlike the four-loop renormalization of QCD, the calculations of moments of structure functions require very considerable computing resources. Much more than thousand times the time of the third-order computation is required at $N=4$, and the scaling of the hardest topologies with $N$ is, at least so far, much worse than that of the MINCER program in its final highly optimized form.

Nevertheless, already now we have been able to calculate enough moments for the determination of the all- $N$ expressions of the $n_{f}^{2}$ contributions to $\gamma_{\mathrm{ns}}^{(3) \pm}$ and the leading large- $n_{f}$ contributions to their flavour singlet counterparts $\gamma_{\mathrm{ik}}^{(3)}$ via Diophantine equations for the coefficients of the harmonic sums. We do not expect that the determination of all- $N$ expressions in this manner can be extended far beyond the point we have reached now. However, we hope to be able to obtain more moments in the future, and to provide approximate results for the $\mathrm{N}^{3} \mathrm{LO}$ splitting functions $P_{\mathrm{ik}}^{(3)}(x)$ that are useful for high-precision calculations of benchmark processes in $e p$ and $p p$ scattering.

\section{Acknowledgements}

This work has been supported by the European Research Council (ERC) Advanced Grant 320651, HEPGAME and the UK Science \& Technology Facilities Council (STFC) grants ST/L000431/1 and ST/K502145/1. Part of our computations were carried out on the Dutch national e-infrastructure with the support of the SURF Cooperative and the PDP Group at Nikhef. We also are grateful for the opportunity to use a substantial part of the ulgqcd computer cluster in Liverpool which was funded by the STFC grant ST/H008837/1. A.V. would like to thank J. Blümlein and A. Grozin for useful discussions during the workshop.

\section{References}

[1] J.M. Campbell, R.K. Ellis and C. Williams, arXiv:1601.00658;

M. Czakon, P. Fiedler, D. Heymes and A. Mitov, JHEP 1605 (2016) 034, arXiv:1601.05375;

R. Boughezal, X. Liu and F. Petriello, arXiv:1602.06965;

J.M. Campbell, R.K. Ellis, Y. Li and C. Williams, arXiv:1603.02663;

M. Grazzini, S. Kallweit, D. Rathlev and M. Wiesemann, arXiv:1604.08576;

M. Grazzini, S. Kallweit, S. Pozzorini, D. Rathlev and M. Wiesemann, arXiv:1605.02716;

A. Gehrmann-De Ridder, T. Gehrmann, E.W.N. Glover, A. Huss and T.A. Morgan, arXiv:1605.04295 
[2] S. Alehkin, J. Blümlein and S. Moch, $\alpha_{s}$ from global fits of parton distribution functions, p. 50-66 in arXiv: 1512.05194

[3] C. Anzai, A. Hasselhuhn, M. Höschele, J. Hoff, W. Kilgore, M. Steinhauser and T. Ueda, JHEP 1507 (2015) 140, arXiv:1506.02674;

C. Anastasiou, C. Duhr, F. Dulat, E. Furlan, T. Gehrmann, F. Herzog, A. Lazopoulos, B. Mistlberger, JHEP 1605 (2016) 058, arXiv:1602.00695

[4] S. Moch, J.A.M. Vermaseren and A. Vogt, Nucl. Phys. B688 (2004) 101, hep-ph/0403192; A. Vogt, S. Moch and J.A.M. Vermaseren, Nucl. Phys. B691 (2004) 129, hep-ph/0404111

[5] T. Ueda, B. Ruijl and J.A.M. Vermaseren, Forcer: a FORM program for four-loop massless progagators, these proceedings; see also arXiv:1604.08767

[6] S.G. Gorishnii, S.A. Larin, L.R. Surguladze, F.V. Tkachov, Comput. Phys. Commun. 55 (1989) 381; S.A. Larin, F.V. Tkachev and J.A.M. Vermaseren, The FORM version of MINCER, NIKHEF-H-91-18

[7] S.A. Larin, T. van Ritbergen and J.A.M. Vermaseren, Nucl. Phys. B427 (1994) 41;

S. Larin, P. Nogueira, T. van Ritbergen, J. Vermaseren, Nucl. Phys. B492 (1997) 338, hep-ph/9605317;

A. Retey and J.A.M. Vermaseren, Nucl. Phys. B604 (2001) 281, hep-ph/0007294

[8] W.L. van Neerven and A. Vogt, Nucl. Phys. B568 (2000) 263, hep-ph/9907472;

W.L. van Neerven and A. Vogt, Nucl. Phys. B588 (2000) 345, hep-ph/0006154;

W.L. van Neerven and A. Vogt, Phys. Lett. B490 (2000) 111, hep-ph/0007362

[9] T. Jaroszewicz, Phys. Lett. B116 (1982) 291;

S. Catani and F. Hautmann, Nucl. Phys. B427 (1994) 475, hep-ph/9405388;

V.S. Fadin and L.N. Lipatov, Phys. Lett. B429 (1998) 127, hep-ph/9802290;

M. Ciafaloni and G. Camici, Phys. Lett. B430, 349 (1998), hep-ph/9803389

[10] J. Blümlein and A. Vogt, Phys. Lett. B370 (1996) 149, hep-ph/9510410

[11] A. Vogt, C.H. Kom, N.A. Lo Presti, G. Soar, A.A. Almasy, S. Moch, J.A.M. Vermaseren, K. Yeats, PoS LL 2012 (2012) 004, arXiv:1212.2932

[12] G.P. Korchemsky, Mod. Phys. Lett. A4 (1989) 1257

[13] Yu. L. Dokshitzer, G. Marchesini and G. P. Salam, Phys. Lett. B634 (2006) 504, hep-ph/0511302

[14] G. Soar, S. Moch, J.A.M. Vermaseren and A. Vogt, Nucl. Phys. B832 (2010) 152, arXiv:0912.0369

[15] A.A. Almasy, G. Soar and A. Vogt, JHEP 1103 (2011) 030, arXiv:1012.3352

[16] S. Moch and M. Rogal, Nucl. Phys. B782 (2007) 51, arXiv:0704.1740

[17] J.F. Bennett and J.A. Gracey, Phys. Lett. B432 (1998) 209, hep-ph/9803446

[18] B. Ruijl, T. Ueda, J.A.M. Vermaseren and A. Vogt, to appear

[19] P.A. Baikov and K.G. Chetyrkin, Nucl. Phys. B (Proc. Suppl.) 160 (2006) 76;

P.A. Baikov, K.G. Chetyrkin, J.H. Kühn, Nucl. Part. Phys. Proc. 261/2 (2015) 3, arXiv:1501.06739

[20] V.N. Velizhanin, Nucl. Phys. B860 (2012) 288, arXiv:1112.3954

[21] V.N. Velizhanin, arXiv:1411.1331

[22] S. Moch, J.A.M. Vermaseren and A. Vogt, Nucl. Phys. B726 (2005) 317, hep-ph/0506288

[23] T. van Ritbergen, J.A.M. Vermaseren and S.A. Larin, Phys. Lett. B400 (1997) 379, hep-ph/9701390 
[24] I. Antoniadis and E. G. Floratos, Nucl. Phys. B191 (1981) 217

[25] A.A. Almasy, S. Moch and A. Vogt, Nucl. Phys. B854 (2012) 133, arXiv:1107.2263

[26] J.A.M. Vermaseren, A. Vogt and S. Moch, Nucl. Phys. B724 (2005) 3, hep-ph/0504242; S. Moch, J.A.M. Vermaseren and A. Vogt, Nucl. Phys. B813 (2009) 220, arXiv:0812.4168 [hep-ph]

[27] I. Bierenbaum, J. Blümlein and S. Klein, Nucl. Phys. B820 (2009) 417, arXiv:0904.3563; H. Kawamura, N.A. Lo Presti, S. Moch and A. Vogt, Nucl. Phys. B864 (2012) 399, arXiv:1205.5727; J. Ablinger, A. Behring, J. Blümlein, A. De Freitas, A. von Manteuffel and C. Schneider, Nucl. Phys. B890 (2014) 48, arXiv:1409.1135;

J. Blümlein, A. De Freitas, C. Schneider, Nucl. Part. Phys. Proc. 261/2 (2015) 185, arXiv:1411.5669

[28] S. Moch and A. Vogt, JHEP 0911 (2009) 099, arXiv:0909.2124;

S. Moch and A. Vogt, JHEP 0904 (2009) 081, arXiv:0902.2342

[29] P.A. Baikov, K.G. Chetyrkin and J.H. Kühn, Phys. Rev. Lett. 104 (2010) 132004, arXiv:1001.3606; P.A. Baikov, K.G. Chetyrkin, J.H. Kühn and J. Rittinger, Phys. Lett. B714 (2012) 62, arXiv:1206.1288

[30] S. Moch, J.A.M. Vermaseren and A. Vogt, Nucl. Phys. B646 (2002) 181, hep-ph/0209100

[31] J.A. Gracey, Phys. Lett. B322 (1994) 141, hep-ph/9401214

[32] J.A.M. Vermaseren, Int. J. Mod. Phys. A14 (1999) 2037, hep-ph/9806280; J. Blümlein and S. Kurth, Phys. Rev. D60 (1999) 014018, hep-ph/9810241

[33] A.K. Lenstra, H.W. Lenstra, Jr., and L. Lovász, Math. Ann. 261 (1982) 515; K. Matthews, Solving $A X=B$ using the Hermite normal form, (unpublished), summarized in J.H. Silverman, Designs, Codes and Cryptography 20 (2000) 5 [see pages 16/17]

[34] V.N. Velizhanin, Nucl. Phys. B864 (2012) 113, arXiv:1203.1022; S. Moch, J.A.M. Vermaseren and A. Vogt, Nucl. Phys. B889 (2014) 351, arXiv:1409.5131

[35] J.Davies, B. Ruijl, T. Ueda, J.A.M. Vermaseren and A. Vogt, to appear

[36] J.M. Henn, A.V. Smirnov, V.A. Smirnov, M. Steinhauser, JHEP 1605 (2016) 066, arXiv:1604.03126v2

[37] A. Grozin, J.M. Henn, G.P. Korchemsky and P. Marquard, JHEP 1601 (2016) 140, arXiv:1510.07803; A. Grozin, Leading and next to leading large $n_{f}$ terms in the cusp anomalous dimension and the quark-antiquark potential, arXiv:1605.03886 (these proceedings)

[38] E. Gardi and L. Magnea, JHEP 0903 (2009) 079, arXiv:0901.1091;

T. Becher and M. Neubert, JHEP 0906 (2009) 081, E: JHEP 1311 (2013) 024, arXiv:0903.1126v5;

E. Gardi and L. Magnea, Nuovo Cim. C32N5-6 (2009) 137, arXiv:0908.3273;

V. Ahrens, M. Neubert and L. Vernazza, JHEP 1209 (2012) 138, arXiv:1208.4847

[39] A.V. Smirnov, V.A. Smirnov and M. Steinhauser, Phys. Lett. B668 (2008) 293, arXiv:0809.1927; C. Anzai, Y. Kiyo and Y. Sumino, Phys. Rev. Lett. 104 (2010) 112003, arXiv:0911.4335

[40] J.A.M. Vermaseren, New features of FORM, math-ph/0010025; M. Tentyukov and J.A.M. Vermaseren, Comput. Phys. Commun. 181 (2010) 1419, hep-ph/0702279; J. Kuipers, T. Ueda, J.A.M. Vermaseren and J. Vollinga, Comput. Phys. Commun. 184 (2013) 1453, arXiv:1203.6543 [cs.SC]

[41] S.A. Larin and J.A M. Vermaseren, Phys. Lett. B259 (1991) 345;

E.B. Zijlstra and W.L. van Neerven, Phys. Lett. B297 (1992) 377;

S.A. Larin, Phys. Lett. B303 (1993) 113, hep-ph/9302240;

S. Moch, J.A.M. Vermaseren and A. Vogt, Phys. Lett. B748 (2015) 432, arXiv:1506.04517 